\title{
ALMOST BOUNDED FUNCTIONS
}

\author{
BY \\ A. W. GOODMAN
}

1. Introduction. Let $\Sigma_{2}$ denote the set of functions of the form

$$
f(z)=a_{1} z+a_{2} z^{2}+\cdots+a_{n} z^{n}+\cdots
$$

which are regular in $|z|<1$ (hereafter denoted by $E$ ) and which satisfy the condition that

$$
f\left(z_{1}\right) f\left(z_{2}\right) \neq 1
$$

for every pair $z_{1}, z_{2}$ in $E$. This class of functions has been the subject of several papers, and quite recently Lebedev and Milin $[8 ; 9]$ proved that for such functions

$$
\left|a_{n}\right| \leqq 1, \quad n=1,2,3, \cdots,
$$

with equality if and only if $f(z)=\eta z^{n},|\eta|=1$. Earlier the inequality (1.3) had been established for $n=1$ by Eilenberg [3], and for $n=2$ by Rogosinski [12], who first raised the question of the validity of (1.3) for all $n$. Both Eilenberg and Rogosinski proved that the additional hypothesis that $f(z)$ be univalent in $E$, originally made by Bieberbach $[1 ; 2]$, was totally unnecessary.

By introducing groups of linear transformations, the condition (1.2) can be made to appear as a special case of a more general condition, and it is this generalization which is initiated and studied in this paper. Indeed if we write

$$
G^{(2)}=\left\{w, \frac{1}{w}\right\}
$$

we have on the one hand a group of linear transformations of order 2; and on the other hand, the condition that, for each $w, f(z)$ assumes in $E$ no more than one value from the set $S^{(2)}(w)=\left\{w, w^{-1}\right\}$ is equivalent to the condition (1.2).

More generally we write

$$
G^{(2 n)}=\left\{L_{1}(w), L_{2}(w), \cdots, L_{2 n}(w)\right\}
$$

for a group of linear transformations

$$
L_{j}(w)=\frac{a_{j} w+b_{j}}{c_{j} w+d_{j}}, \quad a_{j} d_{j}-b_{j} c_{j} \neq 0, \quad j=1,2, \cdots, 2 n,
$$

of even order, and we denote the set of numbers generated by (1.5) for fixed

Presented to the Society, December 29, 1953; received by the editors September 17, 1953. 
$w$ by $S^{(2 n)}(w)=\left\{L_{1}(w), L_{2}(w), \cdots, L_{2 n}(w)\right\}$. With these preparations we have the following:

Definition 1. A function $f(z)$, defined in a region $\Delta$, is said to be almost bounded with respect to the group $G^{(2 n)}$ (A.B. for $G^{(2 n)}$ ) in $\Delta$ if $f(z)$ is meromorphic in $\Delta$, and if for each $w$ ( $\infty$ included) $f(z)$ assumes in $\Delta$ no more than $n$ values from the set $S^{(2 n)}(w)$.

For clarity we remark that $f(z)$ may assume a value arbitrarily often, and still be almost bounded for a group, for example $f(z)=z^{k}$ is A.B. for $G^{(2)}$ in $E$ for every integer $k \neq 0$. On the other hand values in the set $S^{(2 n)}(w)$ are to be counted in accordance with their multiplicity, for example $S^{(2)}(1)=\{1,1\}$ so $f(z)$ must necessarily omit $w=1$ in $\Delta$ if it is to be A.B. for $G^{(2)}$ in $\Delta$.

The primary question is this: to what extent is the inequality (1.3) valid when $f(z)$ is A.B. in $E$ for some group $G^{(2 n)}$ other than $G^{(2)}$ ?

If $K$ is a linear transformation and $K^{-1}$ its inverse, then the transformed set

$$
K G^{(2 n)} K^{-1}=\left\{K L_{j} K^{-1}(w), j=1,2, \cdots, 2 n\right\}
$$

is again a group which may be regarded as equivalent to $G^{(2 n)}$. Since this equivalence class of groups generated by a fixed $G^{(2 n)}$ contains a triple infinity of groups, it is too much to expect that (1.3) will generally be valid, and an example to the contrary will be given in $\$ 4$. The equivalence classes of finite groups of linear transformations are well known (Ford [4, pp. 117-133]) and in $\S 3$ we shall select certain standard forms from each equivalence class. Denoting any one of these standard forms by (S) (see Definition 4), we can then state and prove the following:

THEOREM 1. If $f(z)$ of the form (1.1) is univalent and A.B. for Bs in $E$, then $\left|a_{1}\right| \leqq 1$, with inequality if and only if $f(z) \equiv \eta z,|\eta|=1$.

It is reasonable to conjecture that the condition of univalence is unnecessary and that (1.3) is again valid for all $n$, if $f(z)$ is A.B. for \& in $E$. These conjectures do not appear to be easy to settle. As we shall show in $\$ 4$, the principle of subordination, employed by Eilenberg and Rogosinski to remove the condition of univalence, can not be used here except in a trivial way; and the methods of Lebedev and Milin to prove (1.3) for arbitrary $n$ are also inapplicable. These assertions are proved using a particular group $G_{4}$, and for this reason a detailed study of this group is justified.

2. The group $G_{4}$ and the fundamental theorem. It is obvious that the set of linear transformations

$$
G^{(4)}=\{w, i w,-w,-i w\}
$$

form a group, for which the region $|\arg w|<\pi / 4$ is a fundamental region [4, p. 37]. If this group is transformed as in (1.6) with $K=(w+1) /(w-1)$, the group obtained is 


$$
G_{4}=\left\{w, \frac{w+i}{i w+1}, \frac{1}{w}, \frac{i w+1}{w+i}\right\},
$$

and the transform of the fundamental region is a fundamental region for $G_{4}$ consisting of those points exterior to both of the circles $|w \pm i|^{2}=2$. We take $G_{4}$ as the standard form for the equivalence class generated by (2.1). In general we use subscripts to denote the standard forms for an equivalence class.

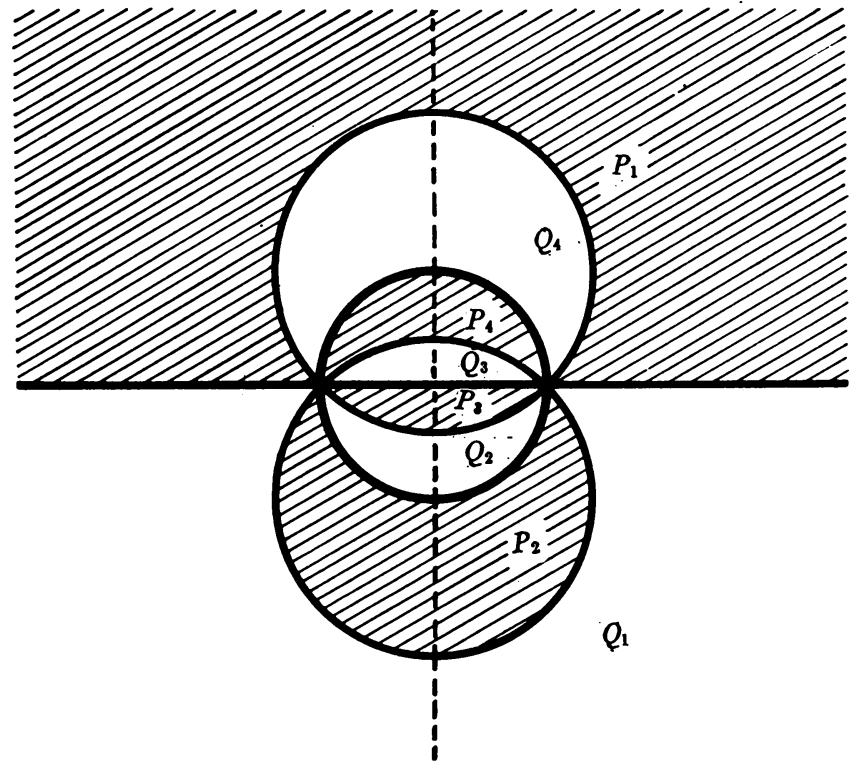

FIG. 1. A set of regions $T$ for $G_{4}$

The fundamental region of $G^{(4)}$ is bisected by the positive real axis, and its image $z^{2}>1$ bisects the fundamental region for $G_{4}$. We denote these two regions by $P_{1}$ and $Q_{1}$, their images by $P_{j}=L_{j}\left(P_{1}\right), Q_{j}=L_{j}\left(Q_{1}\right)$, and we let $T$ denote either a $P$ or a $Q$, or the set of such regions (see Fig. 1). Below is a list of the essential features of this set of regions, in suitable form for any group of even order.

$\mathrm{H}-1$. The number of regions, $4 n$, is twice the order of the group.

H-2. The regions are pairwise disjoint and, except for a finite number of arcs of circles and straight line segments, these regions exhaust the complex plane.

H-3. Each transformation of the group permutes the $P_{j}$ 's and the $Q_{j}$ 's.

H-4. The regions can be divided into two subsets, $T_{j}^{(i)}, j=1,2, \cdots, 2 n$, all of which lie in $E$, and $T_{j}^{(e)}, j=1,2, \cdots, 2 n$, all of which are exterior to $E$.

H-5. For any $j, k=1,2, \cdots, 2 n, A\left(T_{j}^{(e)}\right)>A\left(T_{k}^{(i)}\right)$ where $A(T)$ denotes the area of the set $T$ (possibly infinite). 
H-6. For any fixed $j, L_{k}\left(T_{j}\right), k=1,2, \cdots, 2 n$, forms a set of $2 n$ distinct regions $T$, exactly $n$ of which lie inside $E$.

H-7. The isometric circles $[4$, p. 25] for the transformations of the group are contained in the boundary points of the regions of the set.

For $G_{4}$ the isometric circles are $I_{2}:|w-i|^{2}=2, I_{3}:|w|=1$, and $I_{4}:|w+i|^{2}$ $=2$, so that $\mathrm{H}-7$ is easily checked. In proving $\mathrm{H}-4$, one uses $\mathrm{H}-7$ and the fact that $1 / w$ is an element of the group. The other properties for $G_{4}$ are trivial consequences of the definition of a fundamental region, and the fact that the set of transformations is a group. It should be noted that if $P_{1} \cup Q_{1}$ is contained in a fundamental region (open) for the group, the set $S^{(2 n)}(w)$ will consist of $2 n$ distinct points for each $w$ in $T$.

Definition 2. A group is said to satisfy the conditions $\mathrm{H}$ if a set of regions $T$ can be found satisfying the conditions $\mathrm{H}-1, \cdots, \mathrm{H}-7$.

Definition 3. A point set $F$ is said to be A.B. for $G^{(2 n)}$ if, for each $w$, the set $F$ contains at most $n$ points of the set $S^{(2 n)}(w)$.

Henceforth, we drop the superscripts in $G^{(2 n)}$ and $S^{(2 n)}(w)$, wherever there is no possibility of confusion.

Lemma 1. Let $G$ be a group satisfying the conditions H. If $F$ is A.B. for $G$ and if $F$ contains a neighborhood $N=N\left(w_{0}\right)$ which is contained in some $T$, and if there is a $j$ such that $F$ omits the points $L_{j}(N)$, then the set $F^{\prime}=F-N+L_{j}(N)$ is also A.B. for $G$.

Proof. Since $N$ is contained in some $T, L_{j}(N)$ is contained in $L_{j}(T)$ and it follows from H-2 and H-3 that for each fixed $w, L_{j}(N) \cap S(w)$ is either empty or contains at most one point. If the intersection is empty, then $F^{\prime} \cap S(w)$ $C F \cap S(w)$ and hence $F^{\prime}$ contains at most $n$ points of $S(w)$. If the intersection is not empty, then it contains one point $w^{\prime}=L_{k}(w) \in L_{j}(N)$, and then $F^{\prime}$ must omit $L_{j}^{-1}\left(w^{\prime}\right)=L_{j}^{-1}\left(L_{k}(w)\right)=L_{m}(w) \in N \subset F$. Under these circumstances $F^{\prime} \cap S(w)$ and $F \cap S(w)$ contain the same number of points.

Lemma 2. Let $F_{0}$ be an open set whose closure $F$ is bounded by a finite number of analytic curves, and let $G$ be a group satisfying the conditions $\mathrm{H}$. If $F$ is $A$.B. for $G$, then the complement of $F$ satisfies

$$
A(\Im F) \geqq \pi,
$$

with equality possible only if $\subseteq F=E$.

Proof. The boundary points of the regions $T$ are first deleted from $F_{0}, F$, and $\mathbb{E} F$. By H-7 this includes all isometric circles of the group. The new $F_{0}$ and $F$ still satisfy the conditions of the lemma, if we regard closure as being relative to the complex plane minus the boundary points of the regions $T$. By $\mathrm{H}-2$ the area of $(5 F$ is unchanged.

If $F$ has no points of $E$, then (2.3) is trivial. Further $\mathfrak{S} F$ is open so that if E $F$ contains points not in $E$, then the inequality sign must occur in (2.3). 
Finally in this case the primitive $F$ can not contain boundary points of the regions $T$ in $E$. For with such boundary points $F_{0}$ would also contain points of $E$, so that even after deleting the boundary points of the regions $T$, the new $F$ would still contain points of $E$.

Next suppose $F$ does contain points of $E$. Then $F_{0}$ contains points of $E$, and hence there is a $w_{0}$ which together with some neighborhood $N_{1}$ lies in $F$ and also in some $T_{\boldsymbol{k}}^{(t)}$. By H-6, the set $S\left(w_{0}\right)$ contains $n$ points in $E$ and $n$ points exterior to $E$ and since $F$ is assumed to be A.B. for $G$, at least one of the points $L_{j}\left(w_{0}\right)$ lying outside $E$ is omitted by $F$. But $\subseteq F$ is open so there is a neighborhood $N_{2}$ of $L_{j}\left(w_{0}\right)$ contained in $\subseteq F$. Now $N_{1} \cap L_{j}^{-1}\left(N_{2}\right)$ is open and hence contains a neighborhood $N\left(w_{0}\right)$ satisfying the conditions of Lemma 1. The new set $F^{\prime}$ formed as in Lemma 1 will not be closed, but this can easily be repaired as we shall now show. Since $L_{j}\left(w_{0}\right)$ lies outside $E$ and $w_{0} \in T_{k}^{(i)}$, it follows from H-3 and H-4 that $L_{j}\left(T_{k}^{(i)}\right)=T_{m}^{(e)}$ for some $m$; i.e. it lies outside $E$. Hence by H-5, $A\left(L_{j}\left(T_{k}^{(i)}\right)\right)>A\left(T_{k}^{(i)}\right)$. But from H-7, either $T_{k}^{(i)}$ lies completely inside the isometric circle of $L_{j}$, or completely outside this circle, and the inequality for the areas shows that the former is the case. But since areas inside the isometric circle are magnified by the transformation, it follows that $A(N)<A\left(L_{j}(N)\right)$. We can now select $N_{3}$ so that its closure $\bar{N}_{3} \subset N$, and $A(N)<A\left(L_{j}\left(N_{3}\right)\right)$. Then the new set

$$
F^{(1)}=F-N+L_{j}\left(\bar{N}_{3}\right)
$$

is closed, bounded by a finite number of analytic curves, and is the closure of some open set $F_{0}^{(1)}$. By Lemma $1, F^{(1)}$ is A.B. for $G$ and by construction

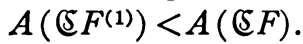

It is clear that (2.4) can be used to generate a sequence of open sets $F_{0}^{(m)}$ with closure $F^{(m)}$, all satisfying the conditions of the lemma, and such that

$$
A\left(\Im F^{(m+1)}\right)<A\left(\Im F^{(m)}\right)
$$

as long as $E \cap F^{(m)}$ is not empty. Further( $\left.{ }^{1}\right)$, for each $\epsilon>0$, there is an $m_{0}$, such that $A\left(E \cap F^{(m)}\right)<\epsilon$ for $m>m_{0}$. For such $m, A\left(\mathbb{S} F^{(m)}\right) \geqq A\left(E \cap\left(\mathbb{E} F^{(m)}\right)\right.$ $>\pi-\epsilon$. If we set $\delta=A(\mathbb{S} F)-A\left(\mathbb{E} F^{(1)}\right)>0$, and then select $\epsilon<\delta$, it follows from (2.5) that

$$
A(\Im F)>\delta+A\left(\Subset F^{(m)}\right)>\pi+\delta-\epsilon>\pi,
$$

by taking $m$ sufficiently large.

Lemma 3. Let $G$ contain the transformation $L=\theta / w,|\theta|=1$. If $f(z)$ is $A$.B. for $G$ in $E$, then $F(z)=\theta / f(1 / z)$ is $A$.B. for $G$ in $|z|>1$.

Proof. Since $L=\theta / w$ is an element of $G$, the set of numbers $\left\{\theta / L_{j}(w)\right.$, $j=1,2, \cdots, 2 n\} \equiv S^{-1}(w)$ is merely a permutation of the set $S(w)$. If in

(1) This is easily proved on the basis of the Vitali covering theorem applied to the squares contained in the neighborhoods $N$. 
$|z|>1, F(z)$ assumes $k$ values in the set $S(w)$, then $\theta / F(z)$ assumes $k$ values in the set $S^{-1}(w)$ and hence $k$ values in its permutation $S(w)$, and $f(z)$ assumes the same $k$ values in $E$.

THEOREM 2. Let $G$ satisfy the conditions $H$, and contain $L=\theta / w,|\theta|=1$. If $f(z)$ of the form (1.1) is univalent and $A . B$. for $G$ in $E$, then $\left|a_{1}\right| \leqq 1$, with equality possible only if $f(z)=\eta z,|\eta|=1$.

REMARK. We note that $f(z)$ is only assumed to be meromorphic and hence it may have a single pole of first order in $E$. In the simpler case of the group $G^{(2)}$, the fact that $f(0)=0$ implies that $f(z)$ must be regular.

Proof. By Lemma 3 the function

$$
F(z)=b z+\sum_{n=0}^{\infty} \frac{b_{n}}{z^{n}}
$$

is A.B. for $G$ in $|z|>1$. For each fixed $r>1$ the image of $|z| \geqq r$ under $F(z)$ is a set $F_{r}$ satisfying the conditions of Lemma 2 . On the other hand, for univalent functions the area of $\mathfrak{S} F_{r}$ is given by the Gronwall-Bieberbach area formula. Hence for each $r>1$

$$
|b|^{2} r^{2}-\sum_{n=1}^{\infty} \frac{n\left|b_{n}\right|^{2}}{r^{2 n}} \geqq 1,
$$

with equality possible only if $\subseteq F_{r}=E$. It is easy to see that the right side of (2.8) is continuous in $r$ for $1 \leqq r<\infty$, and hence

$$
|b|^{2} \geqq 1+\sum_{n=1}^{\infty} n\left|b_{n}\right|^{2}
$$

from which obviously $|b| \geqq 1$ with equality possible only if $F(z)=b z+b_{0}$.

Suppose that $|b|=1$ and $b_{0} \neq 0$, then $F(z)=b z+b_{0}$ is not A.B. for $G$. For if it were, the set $F_{0}:\left|w-b_{0}\right|>1$ could be altered, just as in the proof of Lemma 2 , to a new open set $F_{0}^{\prime}$ with $A\left(\mathfrak{S} F_{0}^{\prime}\right)<A\left(\Im F_{0}\right)=\pi$. The set $F_{0}^{\prime}$ could then be decreased slightly to a closed set without altering the last inequality, and this would then constitute a contradiction to the assertion of Lemma 2 . Hence if $|b|=1$, then $F(z)=b z$, and since $b=\theta / a_{1}$, the proof of Theorem 2 is complete.

3. The extension to other finite groups. All the finite groups of linear transformations are known [4, pp. 117-133]. They are the elliptic cyclic groups, the group of the dihedron, the groups of the regular solids, and their transforms. The cube and the octahedron generate the same group. So also does the dodecahedron and the icosahedron [4, pp. 127-129].

3a. The elliptic cyclic group. If the fixed points are 0 and $\infty$ this group has the form

$$
G^{(2 n)}=\left\{w, \eta w, \cdots, \eta^{2 n-1} w\right\}
$$


where $\eta=e^{\pi / n}$. The set $|\arg w|<\pi / 2 n$ forms a fundamental region for this group. It is bisected by the positive real axis, and it is clear that the rays $w=r \eta^{k / 2}, k=0,1,2, \cdots, 4 n-1$, are permuted by the transformations of (3.1), as well as the sectors which these rays bound, and the transforms of these rays and sectors will be permuted by the transformed group. Since the group is of even order, (3.1) contains $L_{n+1}=\eta^{n} w=-w$, and the set of rays contains the imaginary axis. The transform of the group (3.1) by (1.6) with $K=(w+1) /(w-1)$ gives the group in standard form:

$$
G_{2 n}=\left\{L_{k+1}=\frac{\left(\eta^{k}+1\right) w+\left(\eta^{k}-1\right)}{\left(\eta^{k}-1\right) w+\left(\eta^{k}+1\right)}, k=0,1,2, \cdots, 2 n-1\right\} .
$$

For $n=2$, this is the group $G_{4}$ given by (2.2). The group (3.2) contains $L_{n+1}=1 / w$. The imaginary axis is transformed by $K$ into the unit circle and the $4 n$ sectors go into regions bounded by arcs of circles. Clearly $2 n$ of these regions lie in $E$ and $2 n$ lie exterior to $E$, so if these regions form the set of regions $T$, it is obvious that the conditions $\mathrm{H}$ are satisfied except perhaps for $\mathrm{H}-5$ and H-7. The figure for this group is similar to Fig. 1 except that there are $2 n$ circles (including the real axis) intersecting at $w= \pm 1$ and the angle of intersection of adjacent pairs is $\pi / n$. Except for the real axis these circles are isometric circles for the transformations of the group. For an easy computation shows that the isometric circle for $L_{k+1}$ is $I_{k+1}:|w-i \cot \pi k / 2 n|$ $=|\csc \pi k / 2 n|, k=1,2, \cdots, 2 n-1$. These circles contain the points \pm 1 and $i \cot \pi k / 4 n$, and since the points $0, \infty, \eta^{k / 2}$ go into $-1,1, i \cot (4 n-k) \pi / 4 n$ under $K$, it is clear that the line $w= \pm r \eta^{k / 2}$ goes into $I_{4 n-k+1}(k=2 n+1$, $2 n+2, \cdots, 4 n-1)$, and the real axis goes into itself. Thus the isometric circles are among the circles bounding the regions $T$, and the condition $\mathrm{H}-7$ is satisfied.

It is geometrically obvious that $\mathrm{H}-5$ is satisfied. A rigorous proof may be obtained by noting that the cyclic group is generated by $L_{2}$ and that all of the regions $P$ and $Q$ in the upper half-plane are images of the two regions in $E$, which have the real axis as common boundary, under some power of $L_{2}$. Further all of these regions, except the one with $\infty$ as a boundary point, are contained in the isometric circle of $L_{2}$. It follows that the areas of these regions form an increasing sequence when ordered in accordance with the intersection of the region on the positive imaginary axis. Hence H-5 is satisfied, and the group (3.2) satisfies the conditions of Theorem 2.

$3 \mathrm{~b}$. The dihedral group. This is the group of a fictitious solid consisting of two regular plane polygons of $n$ sides joined along the perimeter. Geometrically the elements of the group correspond to rotations through an angle $2 k \pi / n$ about an axis perpendicular to the face of the polygon, and rotations through an angle $\pi$ about any diameter of the polygon. We take for its counterpart in the complex plane, the group 


$$
G_{2 n}^{*}=\left\{L_{k+1}=\nu^{k} w, L_{n+k+1}=\frac{1}{\nu^{k} w}, k=0,1,2, \cdots, n-1\right\},
$$

where $\nu=e^{2 \pi i / n}=\eta^{2}$, and we designate (3.3) as the standard form.

For this group the set $0<\arg w<2 \pi / n, 0<|w|<1$, forms a fundamental region which we may bisect by the line segment $w=r \eta, 0<r<1$. It is then obvious that all of the conditions $\mathrm{H}$ are satisfied and $G_{2 n}^{*}$ contains $L_{n+1}=1 / w$, so this group also satisfies the conditions of Theorem 2 . The regions in the case $n=3$ are shown in Fig. 2. In this special case the transform of $G_{6}^{*}$ by $K=(w-\nu) /(-\nu w+1)$ is the anharmonic group.

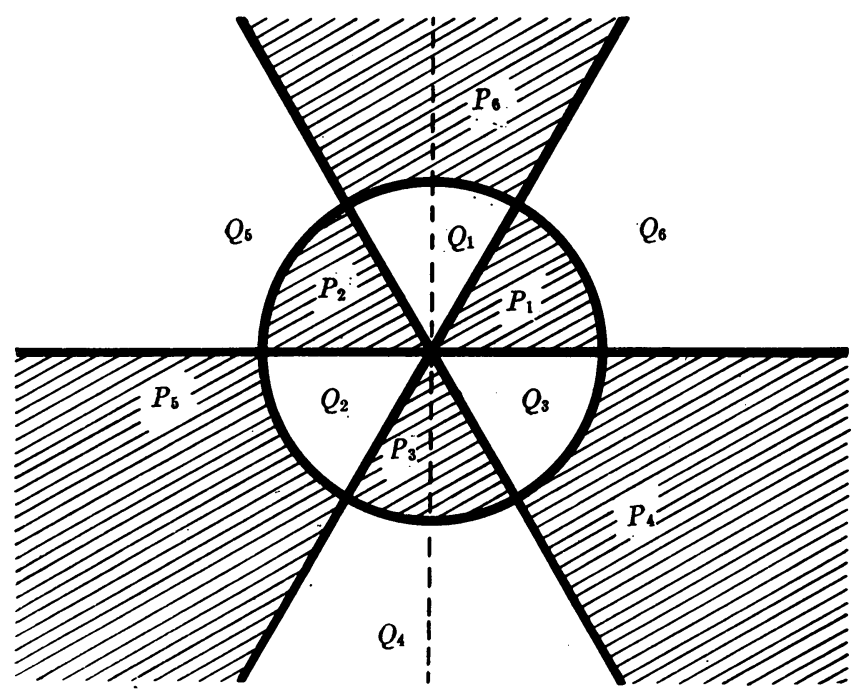

FIG. 2. A set of regions $T$ for $G_{6}{ }^{*}$

REMARKs. It seems clear that for the group $G_{2 n}^{*}$, the process of bisecting a fundamental region is not necessary for the proof of the conclusion of Theorem 2. It is introduced here only in order that this group may be treated simultaneously with the other groups. In case $n=2 m, G_{4 m}^{*}$ can be transformed by $K=(w+1) /(w-1)$ into the group

$$
\begin{aligned}
G_{4 m}^{* *}=\left\{L_{k+1}=\frac{\left(\nu^{k}+1\right) w+\nu^{k}-1}{\left(\nu^{k}-1\right) w+\nu^{k}+1}, L_{2 m+k+1}\right. & =-L_{k+1}, \\
k=0,1,2, \cdots, 2 m-1\}, &
\end{aligned}
$$

which contains $G_{2 m}$ as a subgroup. The circle $|w|=1$ goes into the imaginary axis under $K$, and this merely bisects all of the regions $T$ for the group $G_{2 m}$. It follows that these bisected regions form a suitable set for $G_{4 m}^{* *}$, and hence this group also satisfies the conditions of Theorem 2. A function of the form 
(1.1) which is A.B. for $G_{2 n}^{*}$ must be regular, but it may have a simple pole at $z_{0} \neq 0$ and still be A.B. for $G_{4 m}^{* *}$ (see $\$ 4$ ).

3c. The tetrahedral group. We take as the standard form for this group the set of linear transformations $[7$, p. $42 ; 13$, p. 274]

$$
G_{T}=\left\{ \pm w, \pm \frac{1}{w}, \pm i \frac{w+1}{w-1}, \pm i \frac{w-1}{w+1}, \pm \frac{w+i}{w-i}, \pm \frac{w-i}{w+i}\right\}
$$

It does not appear to be possible to devise a set of regions satisfying the conditions $\mathrm{H}$ for this group. However necessity for such a set can be avoided, by observing that since a tetrahedron can be placed in a cube so that its vertices coincide with a subset of the vertices of the cube, the group $G_{T}$ will be a subgroup of the group of the cube (the octahedral group $G_{0}$ ). To achieve Theorem 2 for this group it is then sufficient to prove the following lemma.

Lemma 4. Let $G_{1}$ be a subgroup of $G$. If a set $F$ is $A$.B. for $G_{1}$, it is $A$.B. for G.

Proof. Let $G_{1}=\left\{L_{1}, \cdots, L_{2 n}\right\}$ have index $k$ in $G$. Just as in the proof of Lagrange's theorem that the order of a subgroup divides the order of a group, we may represent $G$ as the union of $k$ sets of elements $G_{1} L^{(j)}=\left\{L_{1} L^{(j)}, \cdots\right.$, $\left.L_{2 n} L^{(j)}\right\}, j=1,2, \cdots, k$. For any $w, L^{(j)}(w)=w_{j}$, so the set of points generated by $G_{1} L^{(j)}$ for $w$ is identical with the set $S_{1}\left(w_{j}\right)$ and contains at most $n$ points of $F$ by hypothesis. Hence, summing for $j=1,2, \cdots, k$, the set $F$ contains at most $k n$ points of $S(w)$.

As an immediate consequence of Lemma 4, we have the following result, which will be useful later.

LeMmA 5. If the group $G$ contains a transformation of the form $L=\theta / w$, $|\theta|=1$, then $f(z)=\eta z,|\eta|=1$, is $A . B$. for $G$.

3d. The octahedral group. For brevity we list only 6 of the 24 elements of this group. The remaining elements are obtained by replacing $w$ by $i w,-w$, and $-i w$ in turn. The subscripts on $L_{j}$ are to be assigned in such a way that each of the above substitutions increases the subscript by one. With these agreements we have $[7$, p. $43 ; 13$, p. 277$]$

$$
\begin{aligned}
G_{0}=\left\{L_{1}=w, L_{5}=\frac{1}{w}, L_{9}\right. & =i \frac{w+1}{w-1}, \\
& \left.L_{13}=-L_{9}, L_{17}=\frac{w+i}{w-i}, L_{21}=-L_{17}\right\},
\end{aligned}
$$

and the subgroup $G_{T}$ is obtained by taking those elements with odd subscripts.

The isometric circles for this group are the circles $\left|w-i^{m}\right|^{2}=2, m=0,1,2,3$, and $|w|=1$. We can then take for the fundamental region $[4$, p. 77] the region lying in the first quadrant and exterior to all of these circles. This is 
bisected by the line arg $w=\pi / 4$, and the two parts together with their images under elements of $G_{0}$ form a set of 48 regions which are easily found to satisfy the conditions $\mathrm{H}$. The 12 regions of the set $T$ lying in the first quadrant are shown in Fig. 3. The remaining regions are obtained by rotating the figure.

3e. The icosahedral group. We take as the standard form for this group the set $[7$, p. $43 ; 13$, p. 286$]$

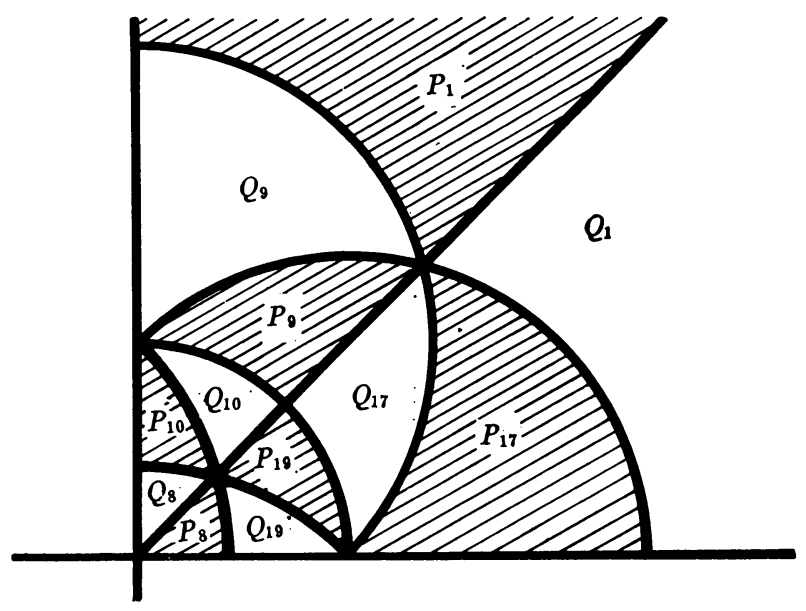

FIG. 3. A set of regions of $T$ for $G_{0}$

$$
G_{I}=\left\{\nu^{n} w,-\frac{\nu^{n}}{w}, \nu^{n} \frac{\nu^{m} \lambda w+1}{\nu^{m} w-\lambda}, \nu^{n} \frac{-\nu^{m} w+\lambda}{\nu^{m} \lambda w+1}, m, n=0,1,2,3,4\right\},
$$

where $\nu=e^{2 \pi i / 5}$ and $\lambda=\left(-1+5^{1 / 2}\right) / 2$. The isometric circles for this group are $|w|=1,|w-\lambda|=\left(10-2 \cdot 5^{1 / 2}\right)^{1 / 2} / 2 \equiv \rho_{1}$, and $\left|w+\lambda^{-1}\right|=\left(10+2 \cdot 5^{1 / 2}\right)^{1 / 2} / 2 \equiv \rho_{2}$ and their images under rotation through an angle $2 \pi m / 5$. Because of the rotational symmetry only the region $0<\arg w<2 \pi / 5$ is shown in Fig. 4 , and our discussion can be restricted to that portion of the plane. The set of points in this sector which lie outside the circle $\left|w+\nu^{3} / \lambda\right|=\rho_{2}$ forms a fundamental region for this group. We bisect this region with the ray $w=r e^{\pi i / 5}$ and the two pieces together with their images form a set of 24 regions $T$ which except for boundary points cover this sector. These regions cannot serve as a set satisfying the conditions $\mathrm{H}$, since 4 of them contain arcs of $|w|=1$ (shown dashed in Fig. 4), and just as in the case of the tetrahedral group, it does not appear to be possible to devise a set of regions for this group which satisfy the conditions $\mathrm{H}$. However by a slight alteration in the proof, the conclusion of Lemma 2 can also be obtained for this group.

Indeed let $E^{*}$ be the points of this sector which lie either in $\left|w-\nu^{2} \lambda^{-1}\right|<\rho_{1}$ or in $\left|w-\nu^{4} \lambda^{-1}\right|<\rho_{1}$. The boundary of $E^{*}$ is shown with heavy lines in Fig. 4. If in the conditions $\mathrm{H}, E$ is replaced by $E^{*}$, then this set of regions obviously 
satisfies all of the conditions except $\mathrm{H}-5$ and $\mathrm{H}-7$. The condition $\mathrm{H}-7$ fails because $|w|=1$ is an isometric circle but is not a part of the boundary of the regions $T$. The condition $\mathrm{H}-5$ may still be valid, the critical regions for comparison being those exceptional regions cut by $|w|=1$, but we do not need to know the areas of these regions.

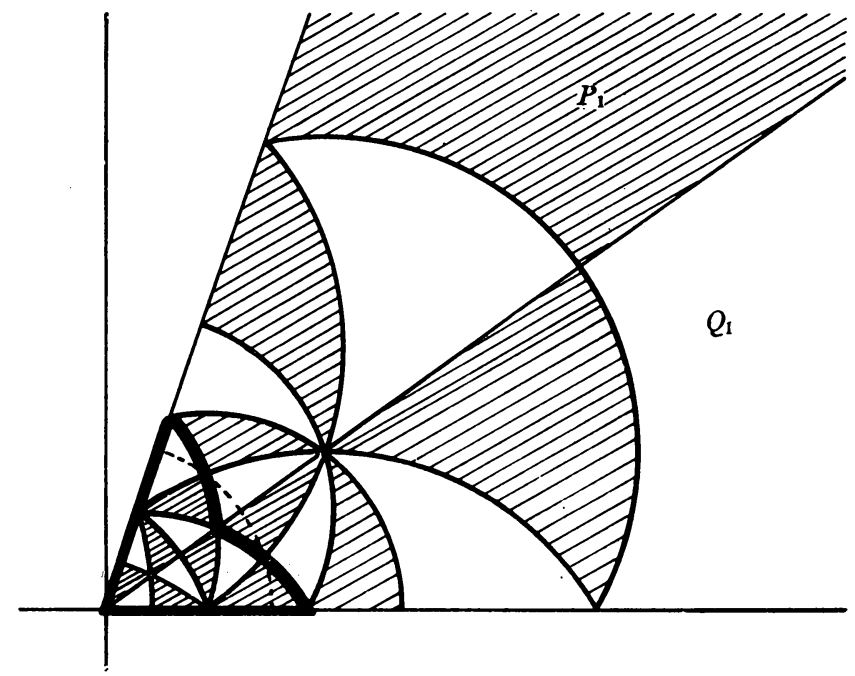

FIG. 4. A set of regions for $G_{I}$

The principal function of the conditions $\mathrm{H}$ was to assure us that in passing from $F$ to $F^{(1)}$ given by (2.4), the inequality (2.5) would be valid. The conditions $\mathrm{H}-5$ and $\mathrm{H}-7$ permitted us to assert that $A(N)<A\left(L_{j}(N)\right)$ for any $L_{j}$ which transformed a neighborhood interior to $E$ into one exterior to $E$, without discussion of the particular transformation involved. Now we must consider three cases. If $N$ lies in a region $T$ completely contained in $E$ and if $L_{j}(T)$ lies completely outside of $E$, then the argument is just as before since $\mathrm{H}-5$ is valid for these regions. If $N$ lies in one of the four exceptional regions cut by $|w|=1$ and $L_{j}(N)$ also lies in one of these exceptional regions, then $L_{j}$ must have the form $-\nu^{n} / w$, and $A(N)<A\left(L_{j}(N)\right)$ is still valid. Finally if one of the two regions $N, L_{j}(N)$ lies in an exceptional $T$ and the other does not, then $L_{j}$ cannot have either the form $\nu^{n} w$, or the form $-\nu^{n} / w$, and again the area must be magnified, since the isometric circle $|w|=1$ no longer plays a part in the argument. Hence Lemma 2 also holds for this group.

Definition 4. By the symbol \&5 we shall mean any one of the groups $G_{2 n}, G_{2 n}^{*}, G_{4 m}^{* *}, G_{T}, G_{0}, G_{I}$ given by (3.2), (3.3), (3.4), (3.5), (3.6), and (3.7) respectively.

Proof of Theorem 1. We have merely to apply Lemma 2 and Theorem 2 to each of the groups symbolized by $(S$. The fact that in each case equality can occur follows from Lemma 5. 
4. Some counter examples. For any constant $c>0$, the set $\{w, c / w\}$ forms a group of linear transformations. Thus if $F$ omits the circle $|w| \leqq c^{1 / 2}$ it is A.B. for this group. Since $c$ is arbitrary, the conclusion of Lemma 2 cannot hold. The function $f(z)=c^{1 / 2} z$ is A.B. for this group in $E$, so the conclusion of Theorem 2 is not valid for arbitrary finite groups. The group may

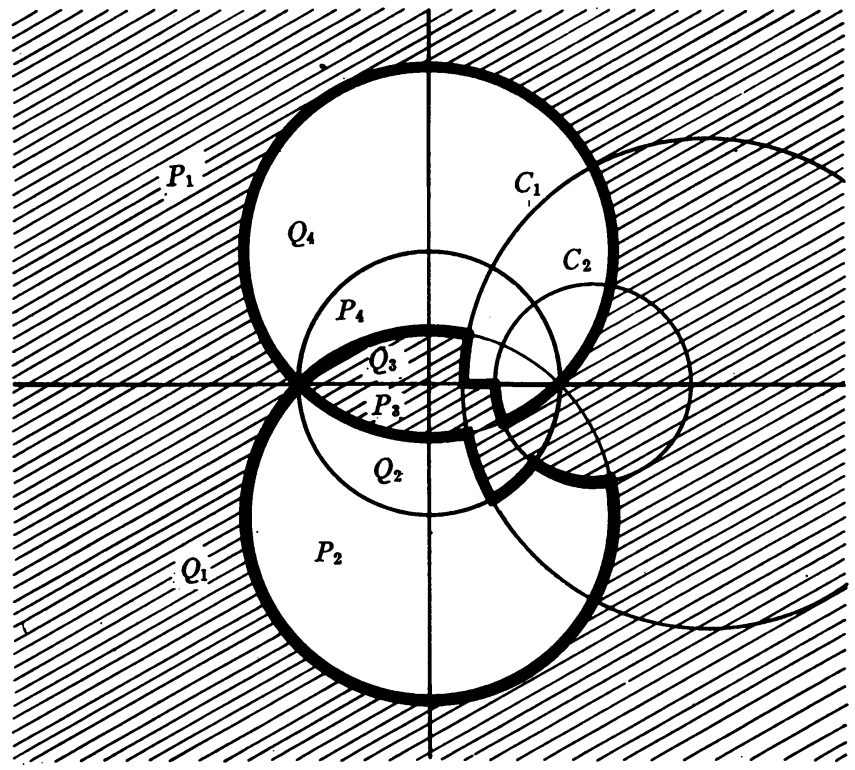

FIG. 5. A set $F$ which is A.B. for $G_{4}$

even contain $1 / w$, but of course it must then be of higher order. For example it can be shown that the function

$$
f(z)=\frac{2 z}{z+3^{1 / 2}}=\sum_{n=1}^{\infty} \frac{2(-1)^{n+1}}{3^{n / 2}} z^{n}, \quad a_{1}=\frac{2}{3^{1 / 2}}>1,
$$

is A.B. in $E$ for the group

$$
\left\{w, \frac{w-2}{2 w-1}, \frac{1}{w}, \frac{2 w-1}{w-2}\right\} .
$$

We next construct a particular set $F$ (see Fig. 5) which is A.B. for $G_{4}$ given by (2.2). If $R$ is a fundamental region for a group, it is sufficient to allow $w$ to run through the points of $\bar{R}$ in order to test whether a set is A.B. for the group. For, any $w$ not in $R$ has the form $w=L_{j}\left(w_{0}\right), w_{0}$ in $\bar{R}$, so that the set $S(w)$ is merely a permutation of the set $S\left(w_{0}\right)$. A set $F$ will thus be A.B. for $G_{4}$ if for each $w$ in $\bar{R}$ the set $F$ contains at most two image points.

Referring to Fig. 1, it is clear that the set $F_{0}$ consisting of the union of 
the sets $P_{1}, Q_{1}, P_{3}, Q_{3}$, and the finite points of the real axis other than \pm 1 is A.B. for $G_{4}$, since $P_{1}, Q_{1}$ and their common boundary points form a fundamental region for this group. Now consider the two circles $C_{1}:|w-17 / 8|$ $=15 / 8$ and $C_{2}:|w-5 / 4|=3 / 4$, of the family orthogonal to the isometric circles of the group. The portions of the regions $T_{j}$ interior to either of these circles are permuted by the transformations of the group. Deleting any such portion and adjoining its image will take an A.B. set into an A.B. set. For $C_{1}$ we delete from $F_{0}$ the portion of $Q_{3}$ and adjoin its image in $Q_{2}$, and for $C_{2}$ we delete the portion of $P_{3}$ and adjoin its image in $P_{2}$. The set so obtained is now made into an open connected set $F$ which is A.B. for $G_{4}$ by deleting appropriate boundary points, and adjoining other boundary points as indicated in Fig. 5. There, the region $F$ is shown shaded and its boundary consists of the heavy solid arcs of circles. The point at $\infty$ is to be regarded as not being in $F$.

In the proof by Lebedev and Milin $\left[8\right.$, p. 395] that $\left|a_{n}\right| \leqq 1$ for functions of the form (1.1), A.B. for $G^{(2)}$ in $E$, essential use is made of the following almost obvious fact.

Lemma 6. Let $s$ be the area of the image of $E$ under $F(z)$ and let $\sigma$ be the area of the set omitted by $F(z)=1 / f(z)$ for $z$ in $E$. If $f(z)$ is univalent and A.B. for $G^{(2)}$ in $E$, then $s \leqq \sigma$.

This lemma can not be extended to arbitrary groups. For if we form the region $F^{*}$, from the region $F$ just constructed, by deleting the points $z<-1$, the new region is simply connected and hence by the Riemann mapping theorem there is a function of the form (1.1) mapping $E$ conformally onto $F^{*}$. Such a function is A.B. for $G_{4}$, and for this function $s=\infty$, while $\sigma$ is finite and so $s>\sigma$. It is clear, then, that the methods of Lebedev and Milin can not be used to extend Theorem 1 to the case $a_{n}, n>1$.

Lemma 7. Let $F$ be a region containing $w=0$, and $A . B$. for $G^{(2)}$. Then there exists a region $f$ which (a) contains $F,(\mathrm{~b})$ is simply connected, and (c) is $A . B$. for $G^{(2)}$.

Using Lemma 7 and the principle of subordination [10], Eilenberg [3], Rogosinski [12], and Lebedev and Milin [8, p. 396] remove the restriction of univalence in the case of $G^{(2)}$. It is again easy to see that these methods do not apply in the general case, since the set $F$ constructed at the beginning of this section is not contained in any simply connected region $f$, unless $f$ is the entire complex plane. But then this $f$ is not A.B. for $G_{4}$. Hence Lemma 7 becomes false if $G^{(2)}$ is replaced by $G_{4}$.

Guelfer [6] and Lohin [11] proved that if $f(z)$ is A.B. for $G^{(2)}$ in $E$, then

$$
\left|f^{\prime}(z)\right| \leqq \frac{\left|1-f^{2}(z)\right|}{1-|z|^{2}},
$$


and the bound is attained for $f(z)=(z+c) /(1+c z)$ when $z$ is real.

This result can not be extended to an arbitrary group $(B)$ as the following considerations show. The transform of $G_{4}^{*}$ under $K=(w+b) /(b w+1)$, $b=-i\left(1+2^{1 / 2}\right)$ is the group

$$
G_{4}^{\prime}=\left\{w, \frac{w-i}{i w-1}, \frac{1}{w}, \frac{i w-1}{w-i}\right\},
$$

which can also be regarded as a subgroup of $G_{0}$. Let $F$ consist of a small open circle about the origin connected with a small open circle about the point $w=1$ by a narrow open strip along the positive real axis. Since $F \cap L_{2}(F)$ and $F \cap L_{4}(F)$ are both empty, $F$ is A.B. for $G_{4}$. Hence if $f(z)$ maps $E$ conformally onto this set, $w=1$ is the image of some point in $E$, while $f^{\prime}(z) \neq 0$ in $E$. Thus (4.3) cannot be valid. If we restrict ourselves to the group $G_{2 n}$, we can establish a number of sharp inequalities including (4.3).

5. The elliptic cyclic group. We need two general lemmas. The first is almost obvious and the proof is omitted.

Lemma 8. If $K=(w+b) /(b w+1), b \neq \pm 1$, then $K G_{2 n} K^{-1}=G_{2 n}$; i.e. the elliptic group given by (3.2) is transformed into itself by $K$.

Lemma 9. If $f(z)$ is A.B. for $G$, then $g(z)=K(f(z))$ is A.B. for $K G K^{-1}$ where $K$ is any linear transformation.

Proof. By hypothesis $f(z)$ assumes at most $n$ values from the set $\left\{L_{j}(w)\right.$, $j=1,2, \cdots, 2 n\}$. Since each $w$ is merely the image of some other $w$ under $K^{-1}, f(z)$ assumes at most $n$ values from the set $\left\{L_{j}\left(K^{-1}(w)\right), j=1,2, \cdots, 2 n\right\}$ for each $w$. Then $K(f(z))$ assumes at most $n$ values from the set

$$
\left\{K\left(L_{j}\left(K^{-1}(w)\right)\right), j=1,2, \cdots, 2 n\right\} .
$$

Theorem 3. Let $f(z)$ be univalent and A.B. for $G_{2 n}$ in E. Then (4.3) is valid, and the equality sign can occur only for functions of the form

$$
f_{e}(z)=\frac{(\eta-\bar{\beta} B) z+B-\eta \beta}{(\eta B-\bar{\beta}) z+(1-B \eta \beta)},
$$

where $|\eta|=1,|\beta|<1, B \neq \pm 1$. If $\beta$ and $B$ are constants subject to these restrictions, then equality occurs in (4.3) at $z=\beta, f_{\theta}(\beta)=B$, and $f_{\theta}(z)$ is $A . B$. for $G_{2 n}$.

Proof. For arbitrary $\beta$ in $E$, set $f(\beta)=B$. Since $f(z)$ is A.B. for an elliptic group, $B \neq \pm 1$. By Lemmas 8 and 9 the function

$$
g(z)=\frac{f\left(\frac{z+\beta}{1+\bar{\beta} z}\right)-B}{1-B f\left(\frac{z+\beta}{1+\bar{\beta} z}\right)}
$$


is univalent and A.B. for $G_{2 n}$ in $E$, and $g(0)=0$. An easy computation yields

$$
g^{\prime}(0)=\frac{f^{\prime}(\beta)\left(1-|\beta|^{2}\right)}{1-f^{2}(\beta)} .
$$

Since $g(z)$ satisfies the conditions of Theorem $1,\left|g^{\prime}(0)\right| \leqq 1$ and (5.3) yields (4.3) at $z=\beta$. Equality can occur only if $g(z)=\eta z$. In this case (5.2) yields

$$
f_{e}\left(\frac{z+\beta}{1+\bar{\beta} z}\right)=\frac{B+\eta z}{1+\eta B z}
$$

which in turn gives (5.1). The function $f_{e}(z)$ maps $|w|=1$ onto a circle containing the points $w= \pm 1$, hence by Lemmas 5,8 , and $9, f_{e}(z)$ is indeed A.B. for $G_{2 n}$ in $E$.

Theorem 4. Let $f(z)=a_{0}+a_{1} z+\cdots$ be univalent and $A . B$. for $G_{2 n}$ in $E$. Then

$$
\left|a_{1}\right| \leqq\left|1-a_{0}^{2}\right|
$$

with equality if and only if

$$
f(z)=\frac{\eta z+a_{0}}{\eta a_{0} z+1}, \quad|\eta|=1 .
$$

Proof. The inequality (5.5) is an immediate consequence of (4.3) with $z=0$. The function (5.6) is obtained from (5.1) by setting $\beta=0$, and $B=a_{0}$.

Theorem 5. If $f(z)=a_{1} z+\cdots$ is univalent and A.B. for $G_{2 n}$ in $E$, then

$$
\begin{gathered}
\frac{1-r}{1+r} \leqq\left|\frac{1+f(z)}{1-f(z)}\right| \leqq \frac{1+r}{1-r} \\
|1-f(z)| \geqq 1-r \\
|1+f(z)| \geqq 1-r
\end{gathered}
$$

where $r=|z|$, and equality can occur only for $f(z) \equiv \eta z$.

Proof. From (4.3), by integrating along a radial line (in the usual manner) we have

$$
\begin{aligned}
\frac{1}{2}\left|\ln \frac{1+f(z)}{1-f(z)}\right| & =\left|\int_{0}^{z} \frac{f^{\prime}(\zeta)}{1-f^{2}(\zeta)} d \zeta\right| \\
& \leqq \int_{0}^{r} \frac{d \rho}{1-\rho^{2}}=\frac{1}{2} \ln \frac{1+r}{1-r}
\end{aligned}
$$

and hence (5.7). Equality can occur in (5.10) only if equality occurs in (4.3) 
at $z=0$. Then, by Theorem $4, f(z)$ must have the form (5.6), and since $a_{0}=0$, $f(z) \equiv \eta z$. To obtain (5.8) note that

$$
\frac{2}{|1-f(z)|}=\left|1+\frac{1+f(z)}{1-f(z)}\right| \leqq 1+\frac{1+r}{1-r}=\frac{2}{1-r},
$$

and for (5.9) note that

$$
\frac{2}{|1+f(z)|}=\left|1+\frac{1-f(z)}{1+f(z)}\right| \leqq 1+\frac{1+r}{1-r}=\frac{2}{1-r} .
$$

These last two theorems were proved earlier by Guelfer [6] for the group $G^{(2)}$.

\section{BIBLIOGRAPHY}

1. L. Bieberbach, Über die Koeffizienten derjeniger Potenzreihen welche eine schlichte Abbildung des Einheitskreises vermitteln, Preuss. Akad. Wiss. Sitzungsber. vol. 38 (1916) pp. 940-955.

2. - Über einige Extremalprobleme in Gebiete der konformen Abbildung, Math. Ann. vol. 77 (1916) pp. 153-172.

3. S. Eilenberg, Sur quelques proprietes topologiques de la surface de sphère, Fund. Math. vol. 25 (1935) pp. 267-272.

4. L. R. Ford, Automorphic functions, New York, McGraw-Hill, 1929.

5. H. Grunsky, Einige Analoga zum Schwarschen Lemma, Math. Ann. vol. 108 (1933) pp. 190-196.

6. S. Guelfer, On the class of regular functions which do not take on any pair of values $w$ and $-w$, Rec. Math. [Mat. Sbornik] N. S. vol. 19 (1946) pp. 33-46.

7. F. Klein, Vorlesungen ibber das Ikosaeder und die Aufösung der Gleichungen fïnften Grades, Leipzig, 1884; English trans., G. G. Morrice, London, 1888; 2d ed., 1913.

8. N. A. Lebedev and I. M. Milin, On the coefficients of certain classes of analytic functions, Doklady Acad. Nauk. SSSR. vol. 67 (1949) pp. 221-223.

9. - On the coefficients of certain classes of analytic functions, Rec. Math. [Mat. Sbornik] N. S. vol. 28 (1951) pp. 359-400.

10. J. E. Littlewood, Lectures on the theory of functions, Oxford University Press, 1944.

11. I. F. Lohin, Remarks on estimates for regular functions, Rec. Math. [Mat. Sbornik] N. S. vol. 24 (1949) pp. 249-262.

12. W. Rogosinski, On a theorem of Bieberbach-Eilenberg, J. London Math. Soc. vol. 14 (1939) pp. 4-11.

13. H. Weber, Lehrbuch der Algebra, vol. II, Braunschweig, 1899.

The University of Kentucky,

LEXINGTON, KY. 